\title{
Commentary on: Economic evaluation of human papilloma virus vaccination in the European Union: a critical review
}

\author{
Gianni Virgili • Giuseppe Turchetti • \\ Roberto Gusinu • Gian Franco Gensini
}

Received: 31 January 2011/Accepted: 14 February 2011/Published online: 9 March 2011

(C) SIMI 2011

The article by Koleva et al. [1] in current issue is unusual reading for the public of IEM. First, systematic reviews of economic evaluations are still uncommon in general medical journals. Second, this review on HPV vaccination deals with a very important public health topic, which, on the other hand, is not a typical theme for this journal.

There are good reasons for the Clinical Evidence and $H T A$ section of IEM to present such articles. Cost effectiveness reviews represent a methodological challenge and, as such, are relevant and intriguing regardless of their object. Furthermore, cost-issues are by their nature a general medical issue, because the cost of resources spent in one field of health care compete with the money spent in others. Finally, an economic publication on a vaccine in a general medical journal is a chance for readers to be challenged by the diverse methodological problems which are encountered in the complex area of screening and vaccines [2].

Coming to Koleva et al.'s message [1], we notice that the authors are critical regarding cost-effectiveness of HPV

G. Virgili ( $\square)$

Department of Specialized Surgical Sciences,

University of Florence and Azienda Ospedaliero-Universitaria

Careggi, V.le Morgagni 85, 50134 Florence, Italy

e-mail: gianni.virgili@unifi.it

G. Turchetti

Istituto di Management, Scuola Superiore Sant'Anna, Pisa, Italy

R. Gusinu

DAI Cardiologico e dei Vasi Azienda Ospedaliero-Universitaria

Careggi, Florence, Italy

G. F. Gensini

Department of Critical Care Medicine and Surgery,

University of Florence and Azienda Ospedaliero-Universitaria

Careggi, Florence, Italy vaccination as measured in the reviewed studies, despite the fact that all but one concluded in favour of vaccination. However, they mention other reviews of economic evaluations which reach similar conservative conclusions [3, 4].They finally observe that vaccination cost-effectiveness was sensitive to the assumptions made in sensitivity analyses and that non-sponsored evaluations were somehow more cautious and restrictive. Koleva et al. [1] are particularly dubious about the validity of life-long modelling of the effects of screening vaccination campaigns, based on a few years follow-up of RCTs and on assumptions borrowed from current data.

The comments raised by Koleva et al. [1] are reasonable. Nevertheless, we have to deal with the limits that we face in performing EEs. In particular, everybody would agree that, for clinical and health policy decisions, it would be desirable to have long term data to work on, rather than referring to assumptions in statistical models. Conditions under which decisions are made are very often different from the desired ones.

Models allow to "rationalize future uncertainty", framing uncertainty within probabilistic models. This is an imperfect method, with large margins of subjectivity, but on the other hand it makes possible-based on more or less optimistic scenarios-to generate forecasts based on epidemiological and clinical data available today, using such predictions to inform decision making. Furthermore, the process is dynamic and it allows to update the estimates by including the evidence provided by new studies.

Regarding the level of subjectivity associated with the selection of model components, if the hypotheses are not "specious", but "reasonable" and "standard/coherent" with the literature $[5,6]$, then the emerging scenario will present a few levels of "controlled and conscious subjectivity". In other words, the limits of the results and the 
caution to be adopted in their interpretation are very well known.

We agree with Koleva et al. [1] that we need to acknowledge the limitations of model-based long term forecasts, but, at the same time, we must be aware of the potential consequences of a "shortermistic" economic approach, which could lead policy makers to have doubts on the efficacy of preventive interventions and strategies, and could discourage the investment in some valuable medium or long term programs. As a result, it may be that the limited budget assigned by many countries to prevention initiatives is also the consequence of this shortermistic vision.

Finally, the whole thing comes down to what rules should we follow for preparing-and who should conduct - the economic evaluations of interventions. The UK National Institute for Clinical Excellence (NICE) has issued guidelines for conducting economic evaluations. NICE also commissions methodological investigations on this issue. The screening setting is of particular complexity, and the HTA report by Karnon et al. [2] is a good example of how sophisticated this type of modelling can be.

The conclusions reached by Koleva et al. [1] make us reflect on a very important question: guidance on methodology for conducting economic evaluations should be issued by European and national governmental health care agencies in collaboration with the international scientific community, since raising costs of health care are likely to have an increasing impact on individual and population health, and may become more and more a potential source of inequality.

Conflict of interest None.

\section{References}

1. Koleva D, De Compadri P, Padula A, Garattini L (2011) Economic evaluation of human papilloma virus vaccination in the European Union: a critical review. Intern Emerg Med. doi:10.1007/s11739011-0529-3

2. Karnon J, Goyder E, Tappenden P, McPhie S, Towers I, Brazier J et al (2007) A review and critique of modelling in prioritising and designing screening programmes. Health Technol Assess 11(52):1-145

3. Newal AT, Beutels P, Wood JG et al (2007) Cost-effectiveness analyses of human papillomavirus vaccination. Lancet Infect Dis 7:289-296

4. Puig-Junoy J, Lopez-Valcarcel BG (2009) Economic evaluations of massive HPV vaccination: within-study and between study variations in incremental cost per QALY gained. Prev Med 48:444-448

5. Brazier J, Ratcliffe J, Salomon JA, Tsuchiya A (2007) Measuring and valuing health benefits for economic evaluation. Oxford University Press, New York

6. Briggs A, Claxton K, Sculpher M (2006) Decision modelling for health economic evaluation. Oxford University Press, New York 